\title{
Ultrafast Structural Phase Transition Driven by Photoinduced Melting of Charge and Orbital Order
}

\author{
P. Beaud, ${ }^{1, *}$ S. L. Johnson, ${ }^{1}$ E. Vorobeva, ${ }^{1}$ U. Staub,${ }^{1}$ R. A. De Souza, ${ }^{1}$ C. J. Milne,${ }^{2}$ Q. X. Jia, ${ }^{3}$ and G. Ingold ${ }^{1}$ \\ ${ }^{1}$ Swiss Light Source, Paul Scherrer Institut, 5232 Villigen PSI, Switzerland \\ ${ }^{2}$ Laboratoire de Spectroscopie Ultrarapide, Ecole Polytechnique Fédérale de Lausanne, 1015 Lausanne, Switzerland \\ ${ }^{3}$ Los Alamos National Laboratory, Los Alamos, New Mexico 87545, USA
}

(Received 7 July 2009; revised manuscript received 14 September 2009; published 9 October 2009)

\begin{abstract}
We use femtosecond $\mathrm{x}$-ray diffraction to probe directly the structural dynamics of a charge ordered and orbitally ordered thin film of $\mathrm{La}_{0.42} \mathrm{Ca}_{0.58} \mathrm{MnO}_{3}$ initiated by an ultrafast optical pulse. At low excitation fluences we observe the displacive excitation of a coherent optical $A_{g}$ phonon. Under high excitation conditions we observe a complete phase transition within $1 \mathrm{ps}$ via the disappearance of a superlattice reflection. The initial step of the phase transition occurs on a time scale significantly faster than the $200 \mathrm{fs}$ time resolution of our experiment.
\end{abstract}

Manganites, transition metal oxides with a perovskitetype structure, exhibit extremely rich phase diagrams that result from a strong coupling among charge, orbital, spin, and lattice degrees of freedom [1]. Both chemical doping and external stimuli such as temperature, magnetic or electric fields can induce very large changes in their macroscopic properties. Of particular interest is the effect of intense pulses of light on these materials [2], which opens up new opportunities to investigate underlying correlations in the time domain [3-10] and even to control material properties on a subpicosecond time scale [3,7-9,11].

The macroscopic properties of the manganites are strongly correlated with the long range order of both the atomic lattice and the electronic system. In ultrafast experiments the properties of the photoexcited system are most often probed via changes in the optical reflectivity. Since the permittivity of a material depends on its structure, coherent optical phonons can often be observed indirectly as oscillations in the measured optical reflectivity [5-7]. Because the permittivity is a complicated function of not only the structure but also the electronic state of the material, it is not possible from measuring the reflectivity alone to deduce unambiguously either the structural or electronic response of the system. Other methods conventionally used to characterize the nature of insulator-metal transitions do not offer sufficient time resolution to investigate ultrafast phase transitions.

Here we apply femtosecond hard x-ray diffraction $[12,13]$ to observe direct and unambiguous structural motion due to laser induced charge- and orbital-order melting in a manganite. Under high excitation conditions we observe the sudden drop of a superlattice (SL) reflection, that exists only in the charge- and orbitally ordered phase. This abrupt decrease of the SL peak intensity is attributed to the immediate release of the Jahn-Teller (J-T) distortion of the oxygen octahedra at the $\mathrm{Mn}^{3+}$ sites [7]. The SL reflection then completely vanishes within 1 ps demonstrating un- equivocally that a change of structural symmetry has occurred on a subpicosecond time scale.

At room temperature $\mathrm{La}_{1-x} \mathrm{Ca}_{x} \mathrm{MnO}_{3}$ (LCMO) is paramagnetic and upon cooling undergoes an insulator-metal transition to a ferromagnetic state for $x$ in the range from 0.17 to 0.5 . For $x>0.5$ the low temperature $(T)$ phase remains insulating with an antiferromagnetic ground state but exhibits charge and orbital order $(\mathrm{CO} / \mathrm{OO})$ [14]. Figure 1(a) illustrates the monoclinic ground state structure of $P 2_{1} / \mathrm{m}$ symmetry as determined for $x=0.5$ by neutron diffraction [15]. The low- $T$ phase is stabilized by the J-T effect that distorts the $\mathrm{O}$ octahedra around the $\mathrm{Mn}^{3+}$ cations. The structural phase transition to the orthorhombic lattice of Pbnm symmetry at $T>T_{\mathrm{CO} / \mathrm{OO}}$ results in a bisection of the unit cell in the direction of the $b$ axis
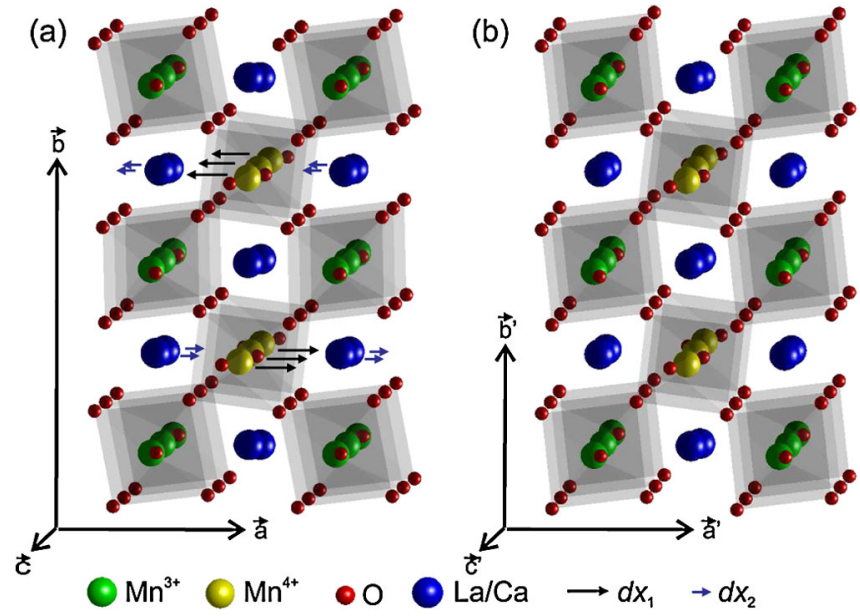

FIG. 1 (color online). (a) Ground state at $20 \mathrm{~K}$ and (b) room temperature structure of half-doped LCMO using the atomic coordinates determined with neutron diffraction [15]. Arrows indicate magnified by a factor 10 the atomic motion in the direction of the $a$ axis predominantly responsible for the change of structural symmetry. 
[see Fig. 1(b)]. Although the forces which drive the phase transition are expected to derive from the J-T effect at the $\mathrm{Mn}^{3+}$ sites, the major structural component of the phase transition within the unit cell can be described in a first order approximation by a shift along the crystal $a$ axis of the $\mathrm{Mn}^{4+}$ (together with the surrounding octahedral $\mathrm{O}_{6}$ cage) by $d x_{1}=0.022$ and of the $\mathrm{La} / \mathrm{Ca}$ atomic positions by $d x_{2}=0.0103$ with respect to the $\mathrm{Mn}^{3+}$ atomic positions [15], where the displacements are expressed in units of the lattice constant $a$.

The (001)-oriented $\mathrm{La}_{0.42} \mathrm{Ca}_{0.58} \mathrm{MnO}_{3}$ film of $55 \mathrm{~nm}$ thickness was grown on a doubleside polished (100) $\mathrm{MgO}$ substrate by pulsed laser deposition. A XeCl excimer laser $(\lambda=308 \mathrm{~nm})$ was used to deposit the films which are maintained at a temperature of $825^{\circ} \mathrm{C}$ under an oxygen pressure of 400 mTorr. $\mathrm{Cu} K \alpha$ radiation was used to check the film crystallinity, orientation, and film thickness. A resistivity versus temperature measurement yielded $T_{\mathrm{CO} / \mathrm{OO}}=235 \mathrm{~K}$, in agreement with the previously published phase diagram of LCMO [14]. The time-resolved diffraction experiments were performed with a grazing incidence geometry [13] using 140 fs x-ray pulses from a tunable synchrotron source [16]. The LCMO film was placed into a vacuum chamber equipped with a large Kapton window allowing the diffracted $\mathrm{x}$ rays to exit the chamber. The film was mounted on a copper block which was brought to a stable temperature of $172 \mathrm{~K}$. A weakly focused $\left(710 \times 720 \mu \mathrm{m}^{2}\right) 100$ fs laser pulse excited the sample with a repetition rate of $1 \mathrm{kHz}$ and a grazing incidence angle of $10^{\circ}$ ( $\pi$ polarization). The x-ray beam (horizontal width $250 \mu \mathrm{m}$ ) was strongly focused vertically to less than $10 \mu \mathrm{m}$. A single $\mathrm{Mo} / \mathrm{B}_{4} \mathrm{C}$ multilayer mirror placed before the sample selected a x-ray energy of $7.15 \mathrm{keV}$ with a bandwidth of $1.2 \%$. The focused horizontally polarized $\mathrm{x}$ rays ( 250 photons/pulse at $2 \mathrm{kHz}$ ) entered the film with vertical grazing incidence of $0.5^{\circ}$ and probed the sample alternately at a time just after the pump pulse ("pumped") and at a time $500 \mu$ s after excitation, when the sample has fully relaxed ("unpumped"). The grazing $\mathrm{x}$-ray incidence angle of $0.5^{\circ}$ yields an attenuation length of the x-ray field of $75 \pm 5 \mathrm{~nm}$ that exceeds the film thickness and the estimated optical penetration depth of the laser of $65 \pm 5 \mathrm{~nm}$ [17].

Our measurements concentrate on the intensity changes in the superlattice (SL) peak derived from the (552) lattice planes in the low- $T$ phase of half-doped LCMO [15]. First we used the regular, "unsliced" monochromatic x-ray beam from a $\mathrm{Si}(111)$ double crystal monochromator to study the temporal evolution of the SL peak with time resolution of 85 ps given by the pulse length of the unsliced electron bunches. The inset of Fig. 2 shows the measured Bragg peak when the sample is rotated with an angle $\phi$ about its surface normal in absence of the optical excitation and when the sample is probed $50 \mathrm{ps}$ after laser excitation at fluences of 2.2 and $4.4 \mathrm{~mJ} / \mathrm{cm}^{2}$. For the higher fluence

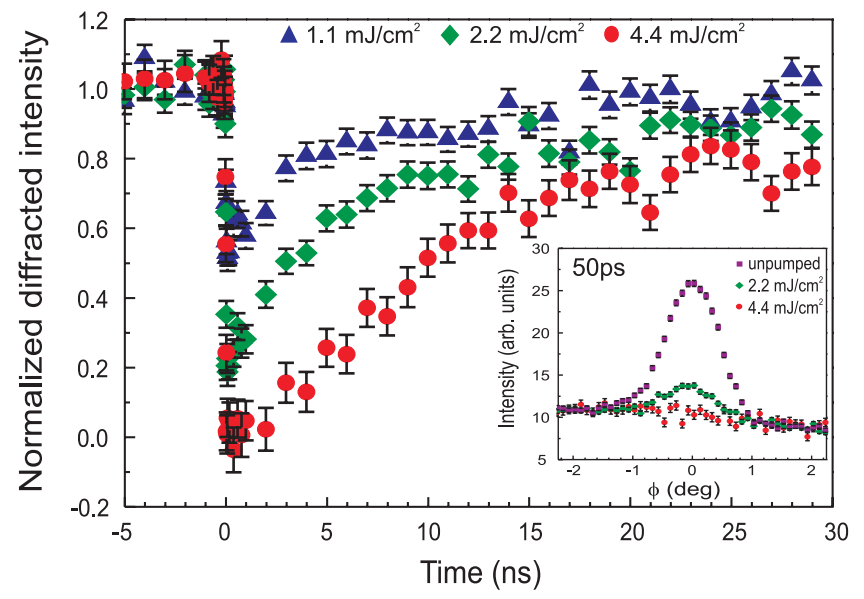

FIG. 2 (color online). Temporal evolution of the SL reflection of the laser excited LCMO film on a nanosecond time scale measured with $90 \mathrm{ps} x$-ray pulses for absorbed laser fluences of $1.1,2.2$, and $4.4 \mathrm{~mJ} / \mathrm{cm}^{2}$, respectively. Inset: Intensity versus $\phi$ measured without and $50 \mathrm{ps}$ after excitation with absorbed laser fluences of 2.2 and $4.4 \mathrm{~mJ} / \mathrm{cm}^{2}$. The background is due to fluorescence from $\mathrm{Mn} K$-edge.

the SL peak has completely vanished, implying that the symmetry of the crystal has increased due to a structural phase transition. Figure 2 shows the normalized diffracted intensity of the SL reflection as a function of time taken at the maximum of the $\phi$ scan for different pump fluences. We observe a disappearance of the SL peak only for a fluence of $4.4 \mathrm{~mJ} / \mathrm{cm}^{2}$ which persists for approximately 2 ns. From the statically measured dependence of the SL peak intensity vs $T$ we estimate that after 50 ps the film temperature has increased to 200 and $225 \mathrm{~K}$ at absorbed (nonreflected) fluences of 1.1 and $2.2 \mathrm{~mJ} / \mathrm{cm}^{2}$, respectively. Using a specific heat of $c_{p}=70 \mathrm{~J} /($ mole K) [18] and averaging the absorbed energy over the film thickness we estimate maximum lattice temperatures of 225 and $280 \mathrm{~K}$ for the two fluences. The lower temperatures inferred from the diffraction data can be explained by loss of energy into the substrate and by the time required for growth of the new phase.

So far we have assumed that the energy absorbed from the laser is converted to heat on the time scale of our measurements. We now turn to the femtosecond time scale, where this assumption breaks down. Figure 3 shows $\phi$ scans of the SL peak taken without excitation, and at pump-probe delay times of $150 \mathrm{fs}$ and 1 ps with a fluence of $7.1 \mathrm{~mJ} / \mathrm{cm}^{2}$. After $150 \mathrm{fs}$ the SL peak drops by $80 \%$ and completely vanishes after $1 \mathrm{ps}$, indicating a fast structural phase transition (PT) triggered by optical excitation.

The time dependence of the SL Bragg reflection for several absorbed pump fluences are summarized in Fig. 4. The data at the lowest fluence of $0.96 \mathrm{~mJ} / \mathrm{cm}^{2}$ show oscillations at approximately $2 \mathrm{THz}$ corresponding to the displacive excitation of a coherent optical phonon that has been observed previously via optical reflectivity 


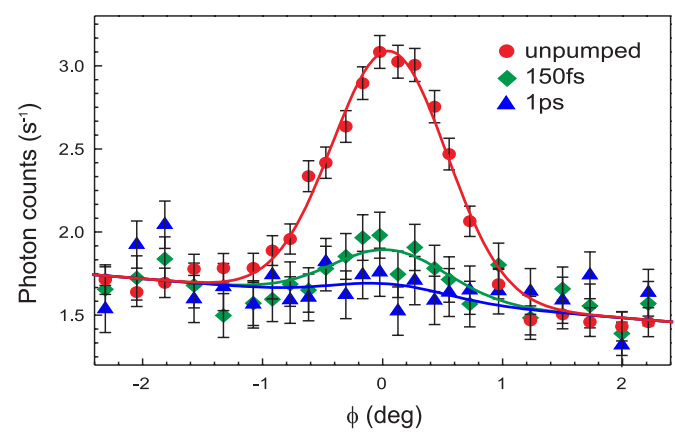

FIG. 3 (color online). $\phi$ scan of the SL reflection without laser, and $150 \mathrm{fs}$, respectively, 1 ps after excitation at an absorbed fluence of $7.1 \mathrm{~mJ} / \mathrm{cm}^{2}$.

measurements [6]. This low frequency $A_{g}$ mode in $R_{1-x} A_{x} \mathrm{MnO}_{3}$ is insensitive to the oxygen isotope but depends strongly on the $A$-cation mass, an observation which has identified this phonon mode with the motion of $\mathrm{La} / \mathrm{Ca}$ atoms in the direction of the crystal $a$ axis [19]. Figure 4(a) shows a fit of the normalized x-ray intensity $I(t) / I_{0}$ to a simple model of displacive excitation [20]:

$$
I(t) / I(0)=1-A e^{-t / \tau_{1}}\left(1-e^{-t / \tau_{2}} \cos 2 \pi \nu t\right),
$$

where $t>0$ is the time and $A$ the phonon amplitude. We account for the experimental temporal resolution of $195 \mathrm{fs}$

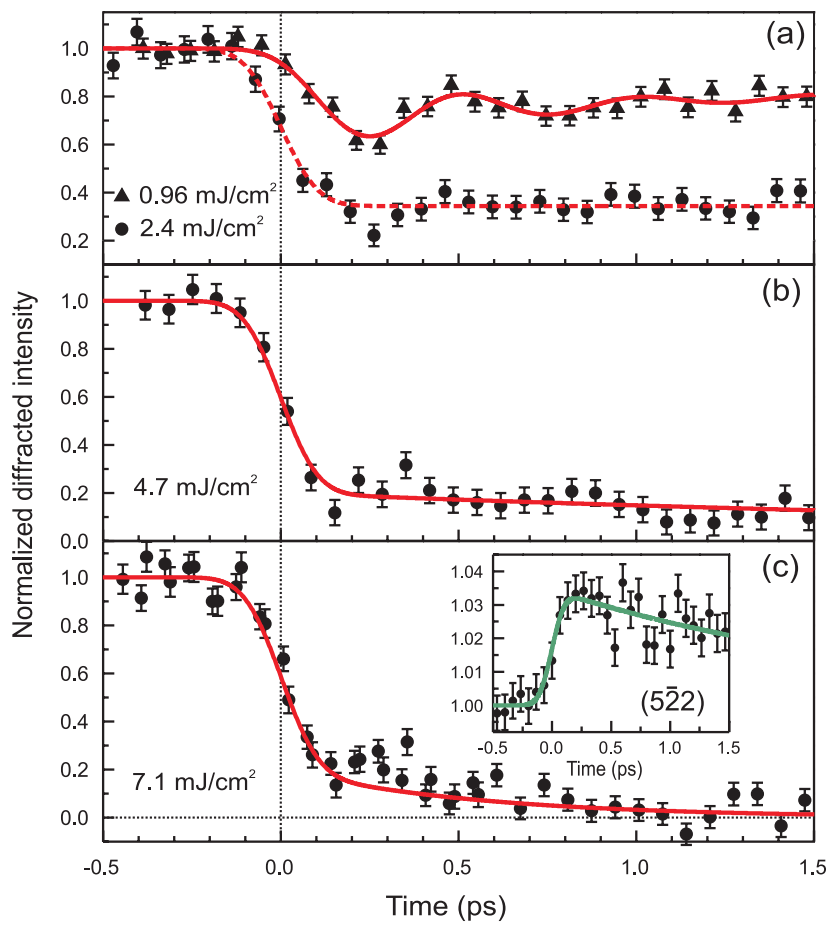

FIG. 4 (color online). Time dependence (a)-(c) of the normalized diffracted intensity of the SL reflection are shown for several pump fluences. Solid curves are fit to the data (see text). Inset: Response of the (52)2) non-SL reflection for an absorbed fluence of $7.1 \mathrm{~mJ} / \mathrm{cm}^{2}$.
[16] by convolution with a Gaussian. The fit yields a phonon frequency $\nu=1.98 \pm 0.12 \mathrm{THz}$, a relaxation time $\tau_{1}=4.5 \pm 1.7 \mathrm{ps}$, and a damping time $\tau_{2}=560 \pm$ $170 \mathrm{fs}$. Assuming that the atomic motion belonging to the observed phonon corresponds to the $d x_{2}$ motion during the PT we can estimate the displacement of the $\mathrm{La} / \mathrm{Ca}$ atoms by calculating the dependence of the $(5 \overline{5} 2)$ reflection intensity on $d x_{2}$ using the atomic coordinates of Ref. [15]. The observed $20 \%$ drop in diffraction intensity corresponds to a laser induced displacement of the $\mathrm{La} / \mathrm{Ca}$ atomic positions by $0.0082(5) \AA$.

For fluences above $4 \mathrm{~mJ} / \mathrm{cm}^{2}$ a significant change of the laser induced dynamics is observed. Independent of pump fluence, the intensity of the SL reflection rapidly drops by approximately $80 \%$ on a time scale faster than the resolution of our experiment. The full structural phase transition involves a $12 \mathrm{pm}$ motion of the $\mathrm{Mn}^{4+}$ sites along the PT coordinate $d x_{1}$, which by itself induces an $80 \%$ drop of the calculated (55) SL peak intensity. We presume that at these excitation levels the J-T distortion at the Mn sites is nearly instantly released. The experimental data in Figs. 4(b) and 4(c) are well described by the solid curves where we assumed an instantaneous drop by $80 \%$ and a subsequential exponential decay, yielding time constants of $3.4 \pm 0.9$ and $0.64 \pm 0.09 \mathrm{ps}$ for the phase transition to complete for excitation fluences of 4.7 and $7.1 \mathrm{~mJ} / \mathrm{cm}^{2}$, respectively. The transient taken at an intermediate fluence of $2.4 \mathrm{~mJ} / \mathrm{cm}^{2}$ in Fig. 4(a) is modeled by a simple step function (dashed curve). Because the laser excitation is inhomogeneous, there may be at this fluence a mixture of regions close to the surface which have undergone the phase transitions and deeper, less excited regions where the excitation does not overcome the PT barrier. To ensure consistency we also measured the dynamics of the non-SL $(5 \overline{2} 2)$ reflection, which during the phase transition should increase by approximately $5 \%$. The measurement [inset in Fig. 4(c)] shows a prompt $3.5 \%$ increase followed by a slow decay which we interpret as onset of laser induced lattice disorder (the Debye-Waller factor) [21].

The time scale for an optically driven structural phase transition is set by the period of phonon modes with the symmetry of the atomic displacement required for the phase transition [22]. For LCMO the modes coupled to charge and orbital order must be those of the oxygen octahedra around the Mn cations. These modes have frequencies in the range of 500 to $600 \mathrm{~cm}^{-1}$ [23], resulting in a temporal limit of 50 to $70 \mathrm{fs}$ for the phase transition which agrees with our experimental result that the first step of the phase transition occurs on a time scale significantly shorter than the experimental resolution of $200 \mathrm{fs}$. The 100 fs pump pulses in our experiment are not short enough to excite such high frequency modes via impulsive stimulated Raman scattering [7]. Hence the atomic motion must be driven by a displacive mechanism following excitation of the electronic system. The photo-absorption mechanism 
of LCMO $(x=0.6)$ for $1.55 \mathrm{eV}$ photons has been attributed mainly to the intra-atomic $t_{2 g}^{3} e_{g}^{1}\left(\mathrm{Mn}^{3+}\right) \rightarrow$ $t_{2 g}^{2} e_{g}^{2}\left(\mathrm{Mn}^{3+}\right)$ transition [17]. Although the electric dipole selection rule forbids such a $d \rightarrow d$ transition, it is optically activated by the strong hybridization of the $\mathrm{Mn} e_{g}$ bands with the $\mathrm{O} 2 p$ states [17]. This transition will promptly introduce local disorder to the orbitals, because it will fill the $\left(3 z^{2}-r^{2}\right)$ orbital and empty an $x y$-type orbital, leading to a different electric quadrupole in the open $3 d$ shell. Because the total electron count at each site in these states remains unchanged, the charge ordering is not directly affected. Rapid electron-electron scattering can, however, quickly thermalize the electronic system and melt the charge order via increased electron hopping between the Mn sites. Such a rapid melting of charge and orbital order would lead to a subsequent release of the J-T induced lattice distortions at the $\mathrm{Mn}^{3+}$ site, driving the motion of the $\mathrm{Mn}^{4+}$ octahedra in the $a$ direction via the shared hybridized $2 p-3 d \mathrm{Mn}-\mathrm{O}$ bonds. The onset of the fast structural reaction coordinate for optical excitation between 0.96 and $2.4 \mathrm{~mJ} / \mathrm{cm}^{2}$ suggests that this threshold is reached once a significant fraction of the $\mathrm{Mn}^{3+}$ ions is excited $\left(2.4 \mathrm{~mJ} / \mathrm{cm}^{2}\right.$ corresponds to the excitation of about $15 \%$ of the $\mathrm{Mn}^{3+}$ averaged over the film thickness). To complete the phase transition the $\mathrm{La} / \mathrm{Ca}$ atoms must adapt to their new equilibrium position. This occurs on a slower time scale due to the weaker Coulomb forces acting on the $\mathrm{La} / \mathrm{Ca}$ cations.

For fluences below $1 \mathrm{~mJ} / \mathrm{cm}^{2}$ our data suggest that the nominal $\mathrm{Mn}^{3+}$ sites remain distorted on a short subpicosecond time scale, but charge and orbital order is only partially disordered. This disordering of the charge and orbital order will cause small changes in the Coulomb field acting on the $\mathrm{La} / \mathrm{Ca}$ sites which could drive the small displacements observed. The further decrease of the SL peak to $50 \%$ after 50 ps (see Fig. 2) indicates a further reduction of the charge and orbital order which we attribute to the thermal generation of coexisting ordered and disordered domains on a picosecond time scale [10]. Finally, the relaxation back to the $\mathrm{CO} / \mathrm{OO}$ ground state is driven by diffusive cooling of the LCMO film via the substrate and occurs on a nanosecond time scale. At pump fluences above the PT threshold the recovery of the ground state phase is slower, due to both the larger heat deposited in the crystal lattice and the first order origin of the $\mathrm{CO} / \mathrm{OO}$ phase transition.

In summary, we have investigated the dynamics of the photoexcited phase transition in LCMO taking advantage of femtosecond x-ray diffraction to probe directly the induced structural dynamics. Above an excitation threshold of approximately $2 \mathrm{~mJ} / \mathrm{cm}^{2}$ the release of the JahnTeller distortion at the $\mathrm{Mn}^{3+}$ sites within less than $100 \mathrm{fs}$ triggers a complete $P 2_{1} / \mathrm{m} \rightarrow \mathrm{Pbnm}$ phase transition. At low excitation fluences the fast increase in disorder of the $\mathrm{CO} / \mathrm{OO}$ phase induces a $<0.01 \AA$ displacement of the $\mathrm{La} / \mathrm{Ca}$ sites thereby exciting the observed $2 \mathrm{THz}$ coherent optical $A_{g}$ phonon mode which previously has also been observed with optical methods [6]. In the near future the several orders of magnitude higher flux of forthcoming free electron laser $\mathrm{x}$-ray sources will help to further clarify the nature of these dynamics by allowing a simultaneous measurement of additional SL reflections with different sensitivity to the motion of the $\mathrm{Mn}$ and $\mathrm{La} / \mathrm{Ca}$ sites with the $\sim 10$ fs resolution required to observe the high frequency structural dynamics associated with the Jahn-Teller related motion of the $\mathrm{Mn}^{3+}$ sites [7]. In addition, time-resolved resonant diffraction will allow a direct measurement of response of the electronic order to photoexcitation [24].

We thank Christof Schneider for helpful discussions, Daniel Grolimund, Camelia Borca, and Alex Oggenfuss for assisting with the experiments. These experiments were performed on the X05LA beam line at the Swiss Light Source, Paul Scherrer Institut, Villigen, Switzerland. The work at Los Alamos was supported by the U.S. Department of Energy through the LANL/LDRD program and the Center for Integrated Nanotechnologies.

*paul.beaud@psi.ch

[1] E. Dagotto, Nanoscale Phase Separation and Colossal Magnetoresistance (Springer-Verlag, Berlin, 2003).

[2] K. Miyano et al., Phys. Rev. Lett. 78, 4257 (1997).

[3] M. Fiebig et al., Appl. Phys. B 71, 211 (2000).

[4] T. Ogasawara et al., Phys. Rev. B 68, 180407(R) (2003).

[5] O. V. Misochko et al., JETP 97, 788 (2003).

[6] D. Lim et al., Phys. Rev. B 71, 134403 (2005).

[7] D. Polli et al., Nature Mater. 6, 643 (2007).

[8] S. Tomimoto et al., Phys. Rev. Lett. 98, 017402 (2007).

[9] M. Matsubara et al., Phys. Rev. Lett. 99, 207401 (2007).

[10] M. Matsubara et al., Phys. Rev. B 77, 094410 (2008).

[11] M. Rini et al., Nature (London) 449, 72 (2007).

[12] A. Cavalleri et al., Phys. Rev. Lett. 87, 237401 (2001).

[13] S. L. Johnson et al., Phys. Rev. Lett. 100, 155501 (2008).

[14] S.-W. Cheong and H. Y. Hwang, in Colossal Magnetoresistance Oxides, edited by Y. Tokura (Gordon and Breach, London, 1999), Chap. 7.

[15] E. E. Rodriguez et al., Phys. Rev. B 71, 104430 (2005).

[16] P. Beaud et al., Phys. Rev. Lett. 99, 174801 (2007).

[17] J. H. Jung et al., Phys. Rev. B 57, R11043 (1998).

[18] C. L. Lu et al., J. Appl. Phys. 103, 07 F714 (2008).

[19] V. A. Amelitchev et al., Phys. Rev. B 63, 104430 (2001).

[20] H. J. Zeiger et al., Phys. Rev. B 45, 768 (1992).

[21] S. L. Johnson et al., Phys. Rev. Lett. 102, 175503 (2009).

[22] A. Cavalleri et al., Phys. Rev. B 70, 161102(R) (2004).

[23] S. Naler et al., Phys. Rev. B 65, 092401 (2002).

[24] G. Ingold et al., Z. Kristallogr. 223, 292 (2008). 